\title{
Inhibition of Anthranilate Synthase Component II, a Novel Protein of $S$. pneumoniae as a Potential Target for Therapy
}

\author{
Mekhala Nagabushan Chitagudigi ${ }^{1}$ (D), Govindaraju Shruthi ${ }^{2}$, Sharanagouda S Patil ${ }^{3}$, Kollur Shiva \\ Prasad ${ }^{4}$, Chandan Shivamallu ${ }^{1, *}$
}

1 Department of Biotechnology and Bioinformatics, School of Life Sciences, JSS Academy of Higher Education \& Research, Mysuru- 570015, India

2 Glimetomics Bioresolve Private Limited, Hootagalli Industrial Area, Mysuru-570018, India

3 ICAR-National Institute of Veterinary Epidemiology and Disease Informatics (NIVEDI), Yelahanka, Bengaluru-560064, India

4 Department of Sciences, Amrita School of Arts and Sciences, Amrita Vishwa Vidyapeetham, Mysuru Campus, Mysuru, Karnataka - 570 026, India

* Correspondence: chandans@jssuni.edu.in (C.S.);

Scopus Author ID 54414904600

Received: 18.03.2021; Revised: 15.06.2021; Accepted: 20.06.2021; Published: 5.09.2021

Abstract: Streptococcus pneumoniae infects the human body primarily through the respiratory tract; however, no evident inflammatory responses are observed upon infection. Even though the inflammatory response is the body's primary immune response, the latency of the inflammatory responses may be attributable to the presence of an anthranilate derivative, quinolone, an isostere of salicylic acid, which acts to suppress inflammation. The reduced immune response promotes the formation of the S. pneumoniae biofilm and increases virulence via quinolone and the derivative, fenamic acid, to elicit different responses. It was found in this study that coumarin binds with good affinity to the binding site of anthranilate synthase component II and also confers a good hemeprotectant property. The enzyme anthranilate synthase is a virulent factor of $S$. pneumoniae and influences the inflammatory response signaling pathways. Inhibition of the anthranilate synthase pathway terminates the virulence of $S$. pneumoniae and helps prevent the impending severe pathogenesis of infection.

Keywords: anthranilate synthase component II; S. pneumoniae; molecular docking; coumarin; virulence.

(C) 2021 by the authors. This article is an open-access article distributed under the terms and conditions of the Creative Commons Attribution (CC BY) license (https://creativecommons.org/licenses/by/4.0/).

\section{Introduction}

Streptococcus pneumoniae was first isolated in 1881, coccus or diplococcus shaped, Gram-positive, facultatively anaerobic bacteria [1]. S. pneumoniae, having over 90 serotypes, is a high-risk pathogenic microorganism that can cause pneumonia, meningitis, otitis media, and septicemia [2]. Of these afflictions, pneumonia is the most common infection of the respiratory tract. Microbial colonization of the nasal and respiratory passages is the primary mode of infection. The symptoms, fever, and coughing, can rapidly transgress from severe to fatal forms of the condition without treatment. It is the most virulent and common cause of community-acquired pneumonia. Nearly $4 \%$ of the Indian population are diagnosed with pneumonia every year, of which 3.6 million cases of severe pneumonia were reported in 
children five years and younger. $16 \%$ of children under the age of 5 worldwide are fatally affected by pneumonia. World Health Organization in 2000 reported 14.5 million severe cases of pneumococcal disease, of which 826,000 deaths resulted in the age group below 18 [2]. The contributing factors for infections include improper treatment, wrong diagnosis, lack of effective and affordable medications [3-7]. Hence, in the current study, the naturally occurring plant component coumarin, which is a potent antimicrobial and antibiofilm moiety, was considered to check on its action against the essential metabolic pathway involving biosynthesis tryptophan [8-14]. Biosynthesis of tryptophan is a necessary pathway for the survival and growth of $S$. pneumoniae [15-17]. The enzymatic components of the tryptophan biosynthesis pathway are also involved in producing several different intermediates such as anthranilate, fenamate which are well-known precursors molecules of nonsteroidal antiinflammatory drugs (NSAIDs) [18-21]. NSAIDs are well-known anti-inflammatory molecules that signal the innate immune response to stop the production of inflammatory molecules in the body [21-23]. The host's production of these moieties is a crucial mechanism involved in immune suppression and immune evasion in the host niche. Therefore, inhibition of anthranilate synthase component II of the tryptophan biosynthesis pathway is targeted using coumarin.

\section{Materials and Methods}

GenBank database was used to analyze S. pneumoniae D39 strain. S. pneumoniae D39 strain is completely annotated with respect to whole-genome assembly. It was found to be virulent and drug-resistant, isolated through clinical samples. Further, the D39 strain of pneumococcus has a complete genome assembly and annotation report, classified up to subspecies level as pneumoniae, and was considered for further studies.

\subsection{Proteome screening.}

The proteome of the selected $S$. pneumoniae strain was procured from the NCBI database. The proteome was further screened for anthranilate synthase component II protein. FASTA sequence of the anthranilate synthase component II protein was procured.

\subsection{Sequence similarity search.}

The FASTA sequence of the anthranilate synthase component II was used to screen the database for proteins with identical sequences across the proteome of the organisms in the nonredundant protein sequence database. BLOSUM 62 matrix was used for the scoring parameter with gap costs comprising 11 for existence and 1 for extension [24,25].

\subsection{Multiple sequence alignment.}

The sequences which were found to be identical to the anthranilate synthase component II protein of S. pneumoniae were considered for multiple sequence alignment to analyze the conserved regions among the sequences. Two independent tools; Multiple sequence alignment of NCBI and MEGA X software, were used [26,27]. Multiple sequence alignment in NCBI was performed using COBALT, using conserved domain and local sequence similarity information among the protein sequences. Alignment parameters were set with gap penalties comprising; gap opening penalty of -11 and gap extension penalty of -1 , end gap opening penalty of -5 , and end gap extension penalty of -1 . 
The similarity sequences of $S$. pneumoniae anthranilate synthase component II protein obtained through BLAST were further subject to multiple sequence alignment using the Clustal $\mathrm{W}$ tool of MEGA X software where the gap opening penalty of 10, gap extension penalty of 0.10 and 0.20 were applied for pairwise alignment and multiple alignments, respectively. The protein weight matrix was set to Gonnet, and residue-specific penalties, hydrophilic penalties were included with a gap separation matrix of $4[27,28]$.

\subsection{Phylogenetic analysis.}

The phylogenetic analysis of the multiple-aligned sequences was carried out through the NCBI COBALT tool and MEGAX software. The phylogenetic tree was generated through the fast minimum evolution method with a maximum sequence difference set to 0.85 . Distance approximation was calculated using the Grishin model, using COBALT.

The multiple-aligned sequences were subject to phylogenetic analysis through the Maximum likelihood method using MEGA X software. The Jone-Taylor-Thornton (JTT) model was used with uniform rates. Tree inference options were set to Nearest-NeighborInterchange(NNI) for ML Heuristic method, and the initial tree was generated through the Neighbor-joining method [29,30].

\subsection{Structural annotation of protein.}

\subsubsection{Homology modeling.}

The FASTA sequence of the anthranilate synthase component II protein was used to predict the protein structure through SwissModel Workspace. A template search for the input sequence was carried out using BLAST and HHblits using the Swiss model template library. Global model quality estimate (GMQE) and quaternary structure quality estimate (QSQE) were used to rank the templates based on the quality. The top-ranked templates were further used to build the model through an open structure computational structural biology framework. The generated models were scored using QMEAN. Additionally, according to the Ramachandran plot, the models generated were assessed with respect to their percentage query cover match and their structural validity.

\subsubsection{Structural validation.}

The generated protein structure models were further scrutinized based on their structural validity. Ramachandran plot of each model was generated using the MolProbity tool to check the accuracy of the generated models. The model with the highest number of residues in favorable and allowed regions was considered. Inferring the selected model exhibited the least number of residues in the disallowed region compared to the rest of the models analyzed. The quality of the protein structures was checked based on their z-score using ProSA server[31,32].

\subsubsection{Protein structure refinement.}

The selected model was subject to structural refinement to correct the structural errors in the residues found in the disallowed region of the Ramachandran plot. The structural refinement of the selected protein was carried out using the GalaxyRefine tool. The protein 
model was subject to structural perturbation using the triaxial loop closure method, and overall relaxation was carried out through molecular dynamic simulation [33].

\subsubsection{Validation of refined structure.}

MolProbity tool was used to predict the structural validity of the predicted structure. The percentage of residues in the favorable region, allowed region, and disallowed region of the Ramachandran plot was assessed to check if the residues with disallowed geometry were corrected. The quality of the purified protein structure was checked based on a z-score using the ProSA server [31,32].

\subsubsection{Binding site prediction.}

Binding site prediction was performed to analyze the probable ligand binding sites in the refined anthranilate synthase component II protein structure. Galaxy Site tool was used to predict ligand-binding sites in the protein through $\mathrm{HH}$ Search. The binding site of the structurally and functionally identical proteins procured from PDB was analyzed through ligand explorer from RCSB PDB. The reported ligand was considered for molecular interaction studies to analyze the binding affinity of coumarin to the protein binding site[33].

\section{2..6. Molecular docking studies.}

Molecular interaction studies of the ligand were carried out to check the validity of the proposed function of the protein. The binding affinity and interactions in the binding sites were considered final functional annotations for the structurally annotated protein. The binding interaction and affinity of the ligand with structurally annotated protein were compared with proteins from other genera in the database for structurally and functionally identical.

\subsection{Ligand preparation.}

The 2D structure of coumarin was sketched using the ACS chemsketch tool[34]. The 2D structure was cleaned for proper geometrical alignment. The addition of 3D coordinates generated the 3D structure of the ligands. The geometry of the 3D structure was cleaned using the Argus lab tool. The refined 3D structure of the ligands was used for molecular docking studies.

Molecular interaction studies were carried out using the Autodock4 tool. The gridbox was set to the predicted binding site of the structurally annotated protein.

The procured crystal structure of the protein from RCSB PDB was further refined by removing water residues, followed by the addition of gasteiger charges, and was further refined by merging non-polar hydrogens using Autodock V.4.0 [35-37].

\subsection{Autogrid generation.}

Upon refinement of protein, the protein structure was selected for rigid molecule, and the 3D structure of the ligand was selected for map type. Based on the binding site residues, the grid box was set, where all the binding site residues fit inside the grid box, and hence the grid box was set with X:16.678 $\mathrm{A}, \mathrm{Y}: 46.384 \AA$, and Z:18.095 $\AA$ dimensions. Upon saving the grid, the grid parameter file (GPF) was generated and saved. The saved grid parameter file was used to run using Autogrid4 [36]. 
On successful completion of autogrid molecular docking of the ligand was carried out using genetic algorithm using Autodock4, followed by generation of docking parameter file (DPF). The saved docking parameter file was used to run Autodock4.

\subsection{Interaction analysis.}

Various complexes of protein-ligand interactions generated from molecular docking studies were analyzed using the UCSF chimera tool. The complexes that exhibited higher interactions with high binding affinity were selected [38].

\subsection{Toxicity profile of coumarin.}

The toxicity of coumarin was analyzed through ADMET studies using the swiss ADME tool, followed by in vitro toxicity analysis through hemolytic assay [39-41].

\subsection{Hemolytic assay.}

Blood from healthy volunteers who were not under NSAIDs was collected in heparin tubes, and the RBC was separated by centrifugation at $3000 \mathrm{rpm}$ for 10 minutes at four ${ }^{\circ} \mathrm{C}$. The $\mathrm{RBC}$ was suspended in Phosphate buffer saline $\mathrm{pH} 7.4$ to obtain 2\% suspension. Triton X100 was used as a positive control for hemolysis, PBS was used as a negative control. $10 \mathrm{mg}$ of coumarin and gentamycin respectively were used to prevent hemolysis where the RBCs were allowed to be infected by $S$. pneumoniae. $50 \mu \mathrm{l}$ of RBC was taken in each tube, $20 \mu \mathrm{l}$ of $S$. pneumoniae suspension was added to each tube except for positive control, and negative control and was incubated at $37^{\circ} \mathrm{C}$ for 30 minutes, followed by centrifugation at $3000 \mathrm{rpm}$ for 10 minutes at $4^{\circ} \mathrm{C}$ to check the formation of RBC pellet. The absence of pellet indicates $100 \%$ hemolysis [42].

\section{Results and Discussion}

The sequence of anthranilate synthase component II was found to have 188 amino acid residues in its sequence. WP_000601926.1 is the protein product of anthranilate synthase component II in the $S$. pneumoniae-D39 strain.

\subsection{Similarity search.}

Upon sequence similarity search of anthranilate synthase component II it was found that the protein was $99 \%$ identical to 33 other $S$. pneumoniae anthranilate synthase component II sequences, $98 \%$ similar to 65 sequences of anthranilate synthase component II from various S. pneumoniae strains, and $97 \%$ identical to the anthranilate synthase component II sequence of $S$. mitis with $100 \%$ query cover with all the 100 similar sequences.

\subsection{Multiple sequence alignment.}

S. pneumoniae anthranilate synthase component II was found to have $69.68 \%$ of the sequence conserved, with the overall mean distance among the sequences found to be 0.02 . Further, S. pneumoniae-D39 anthranilate synthase component II was found to be identical to anthranilate synthase component II of other bacterial species such as P. aeruginosa and Lactococcus. 


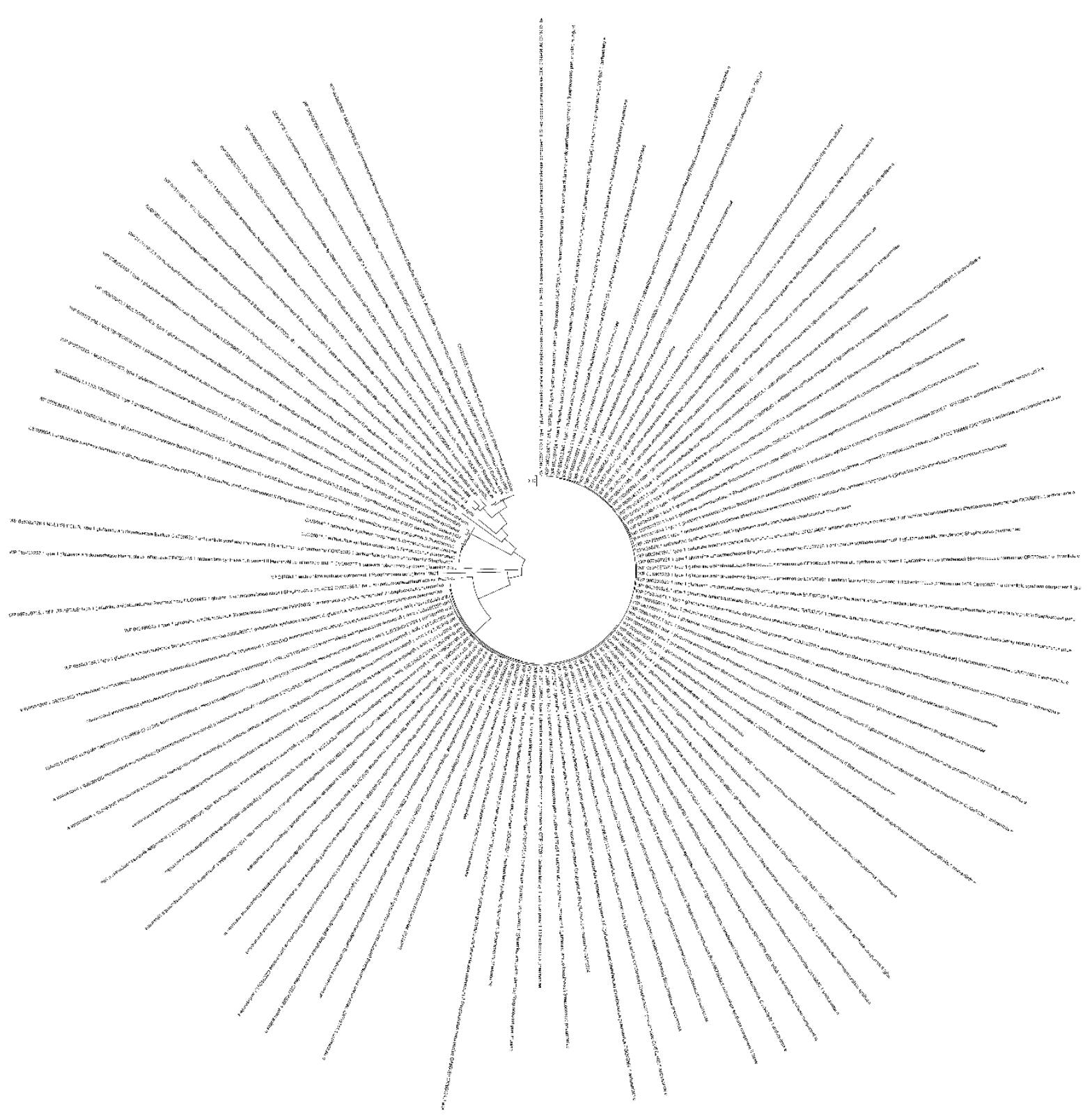

Figure 1. Phylogenetic tree of S. pneumoniae anthranilate synthase component II with a mean distance of 0.02 .

\subsection{Structural elucidation of anthranilate synthase component II.}

The secondary structure of the protein predicted through the PSIPRED server had seven helices, ten strands interspersed with coils. The quarternary structure of the protein predicted through the SwissModel workspace had good quality with $91.85 \%$ of the residues in the Ramachandran favored region, $1.63 \%$ residues in the outlier region and the rest $6.5 \%$ in the allowed region. Hence, the structure was considered for further studies. Upon refinement, the selected structure resulted in a more stereochemically accepted model with $96.25 \%$ of the residues in the Ramachandran favored region and $3.4 \%$ of the residues in the allowed region, and only $0.37 \%$ residues in the outlier region. Hence, the structure was considered fit for molecular interaction studies with coumarin. The structure of anthranilate synthase component II obtained through the swiss model workspace was found to be a homodimer 


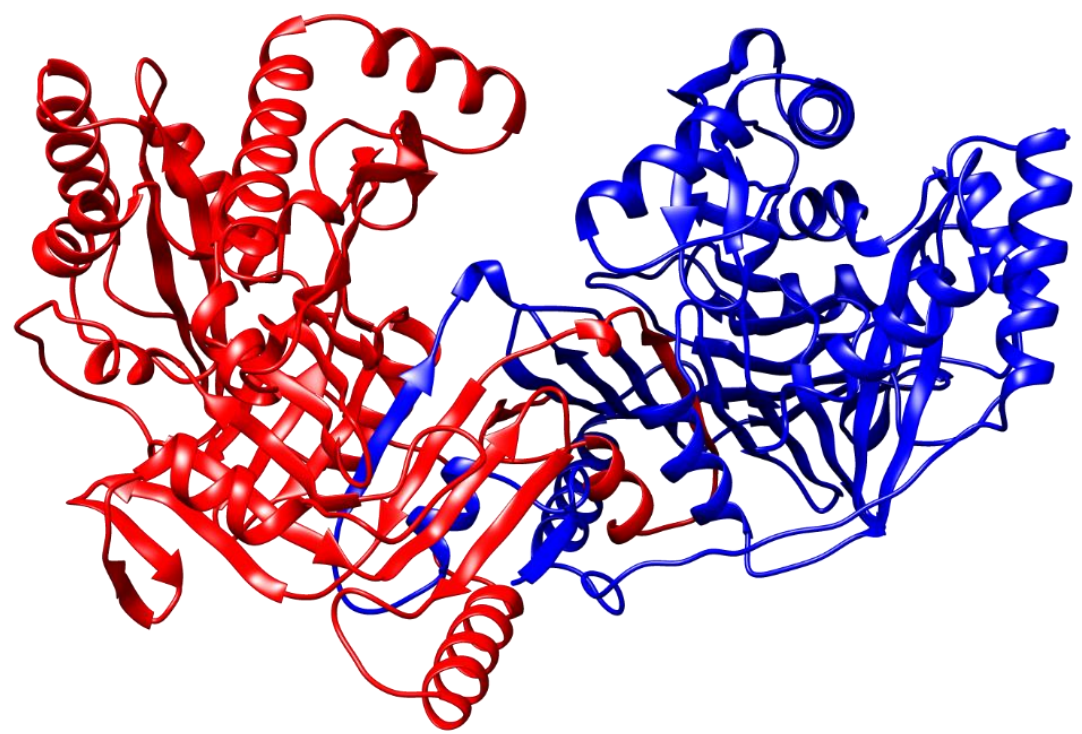

Figure 2. Quaternary structure of $S$. pneumoniae anthranilate synthase component II exhibiting homodimer predicted through SwissModel Workspace.

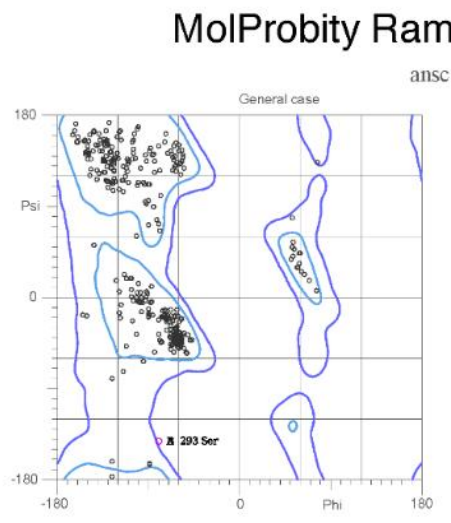

analysis
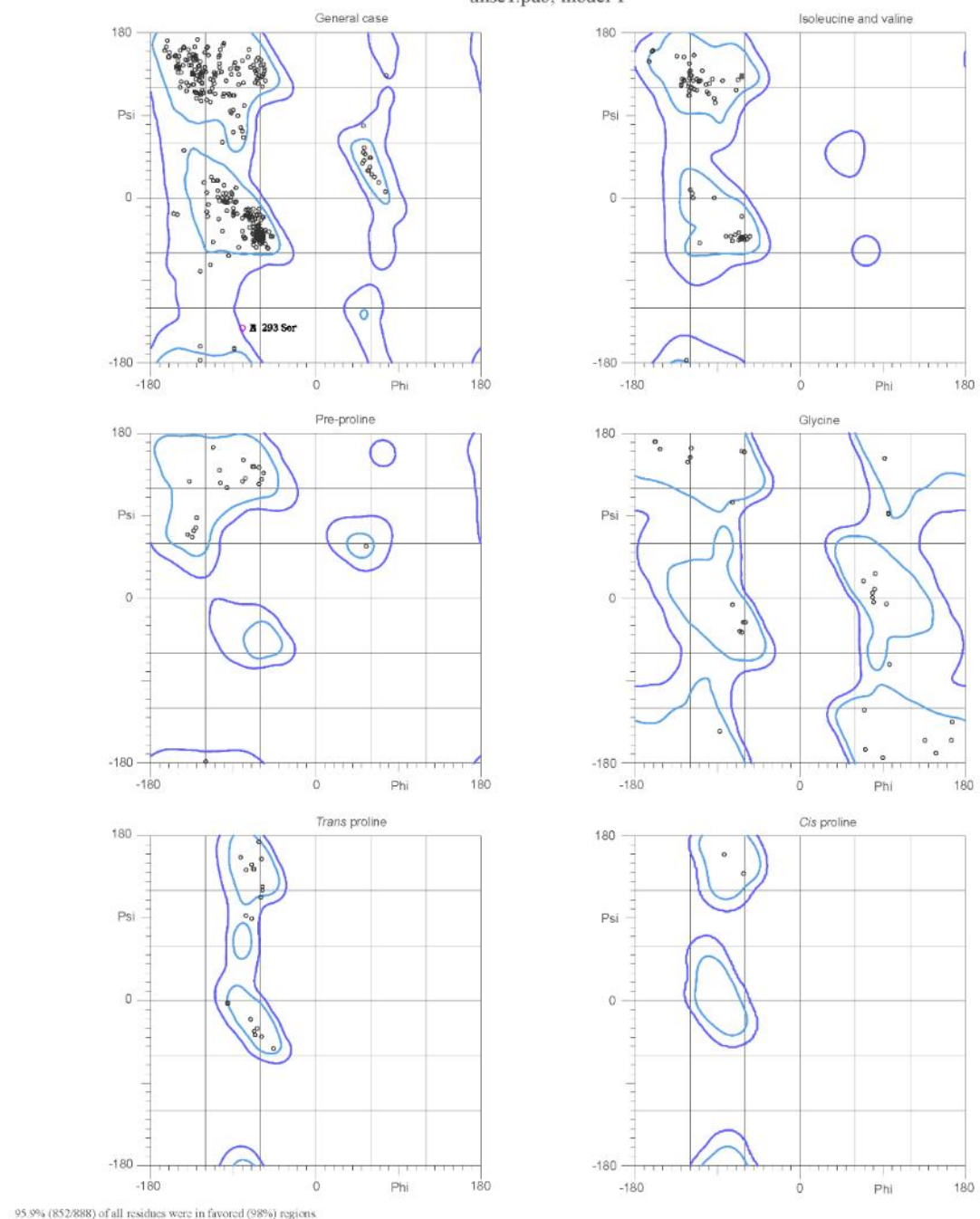

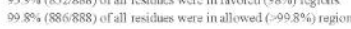

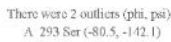

Figure 3. Ramachandran plot of the refined structure of $S$. pneumoniae anthranilate synthase component II, predicted through MolProbity. 


\subsection{Binding site prediction.}

The binding site of TRP in anthranilate synthase component II was constituted by the amino acid residues PHE39, ASN238, TYR239, LEU240, TYR241, ILE392, GLY393, and TYR394.

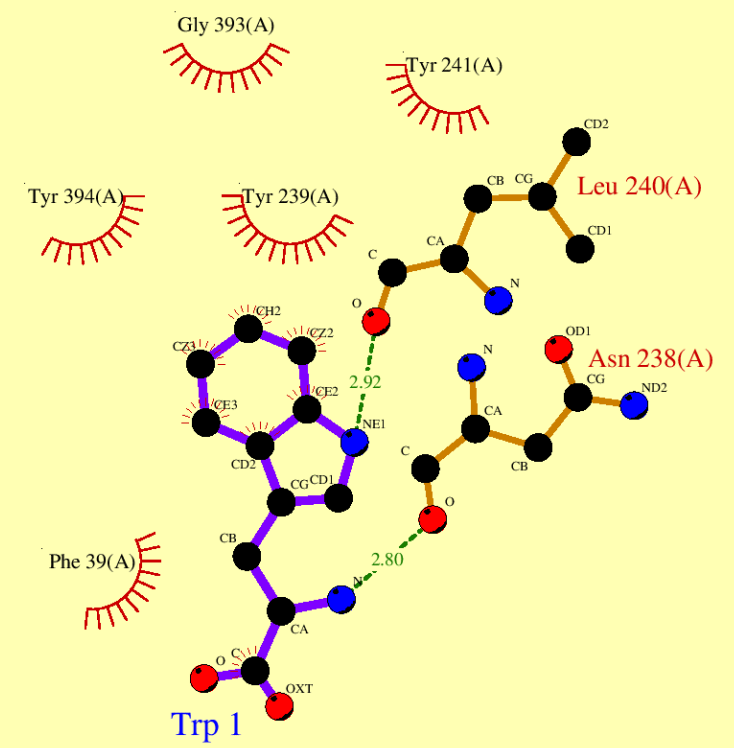

Key

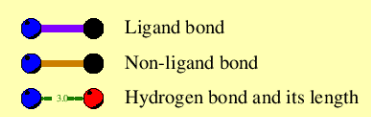

$$
\begin{aligned}
& \text { His } 53 \\
& \eta_{\pi} \text { Non-ligand residues involved in hydrophobic } \\
& \text { contact(s) }
\end{aligned}
$$

Corresponding atoms involved in hydrophobic contact(s)

Figure 4. Binding site of S. pneumoniae anthranilate synthase component II bound by its substrate/ligand, tryptophan.

\subsection{Molecular interaction studies.}

The coumarin molecule was found to interact with the binding site of anthranilate synthase component II with hydrogen bond interaction with LEU240, and hydrophobic interaction with TYR239, TYR394, LEU473, GLU474, and ASP402, with a binding energy of $-7.70 \mathrm{kcal} / \mathrm{mol}$.

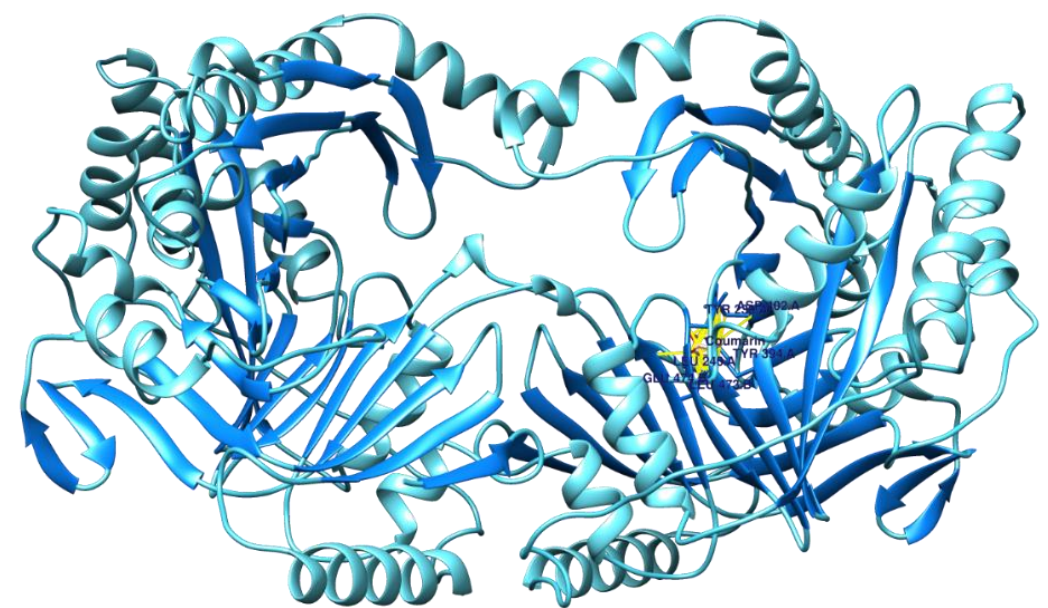

Figure 5. Coumarin bound to the binding site of anthranilate synthase component II, ribbon model. 


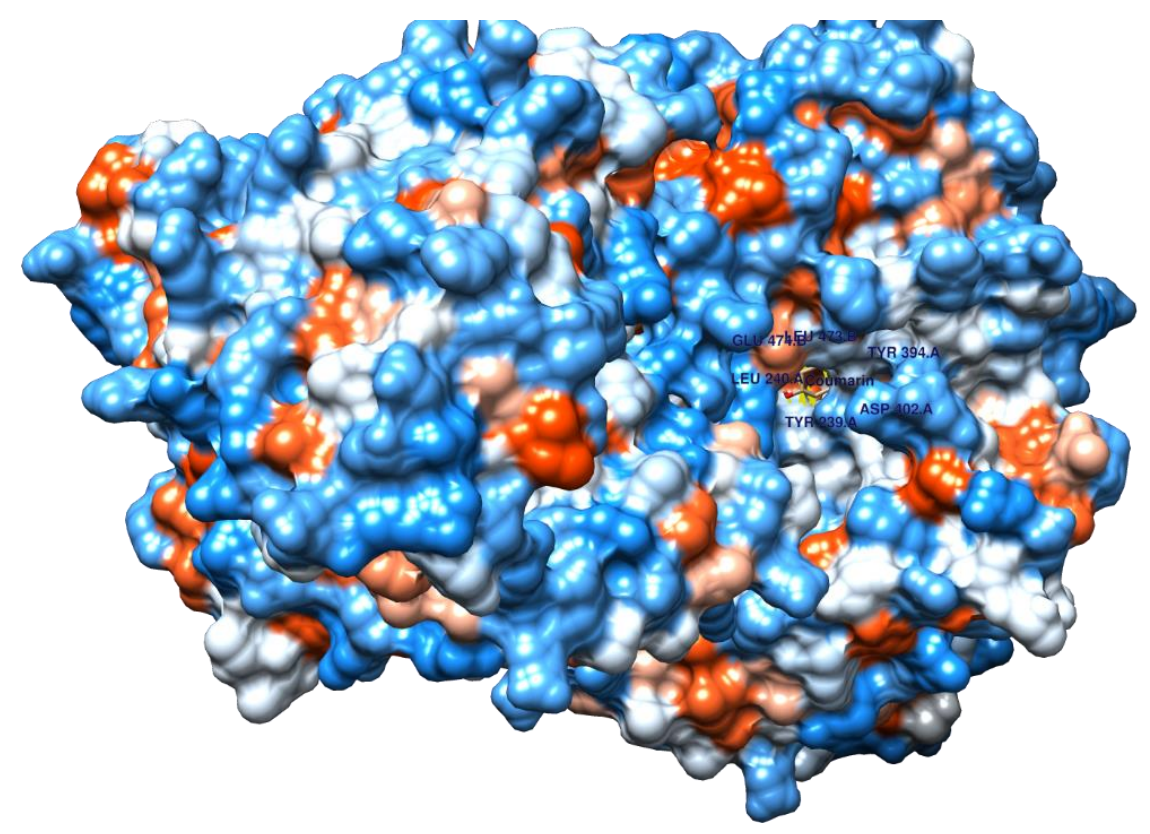

Figure 6. Interaction of coumarin at the binding site of anthranilate synthase component II, hydrophobicity surface model.

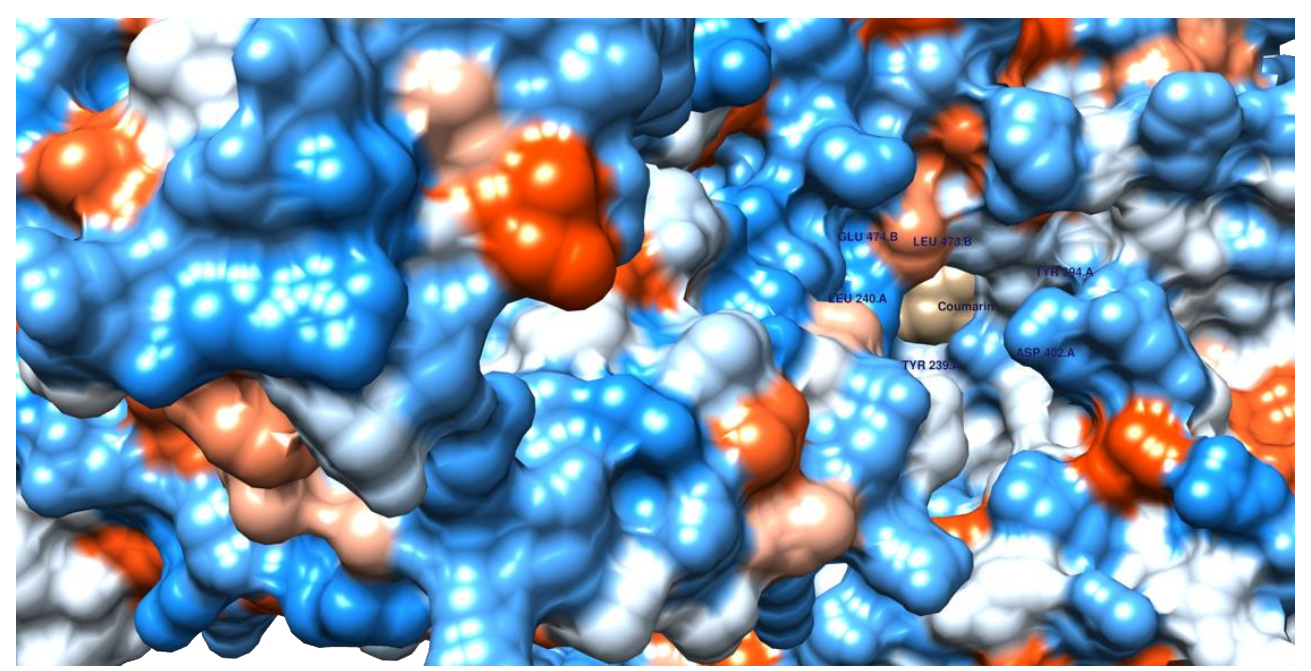

Figure 7. Hydrophobicity surface model of coumarin in the binding pocket of anthranilate synthase component II, hydrophobicity surface model.

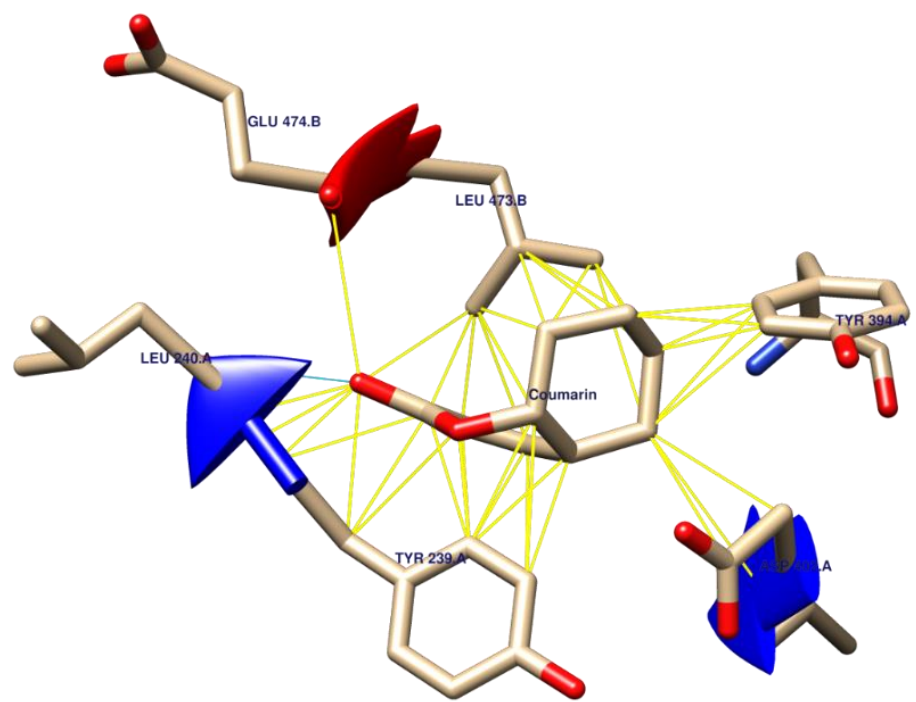

Figure 8. Interaction of coumarin with the binding site residues of anthranilate synthase component II. 


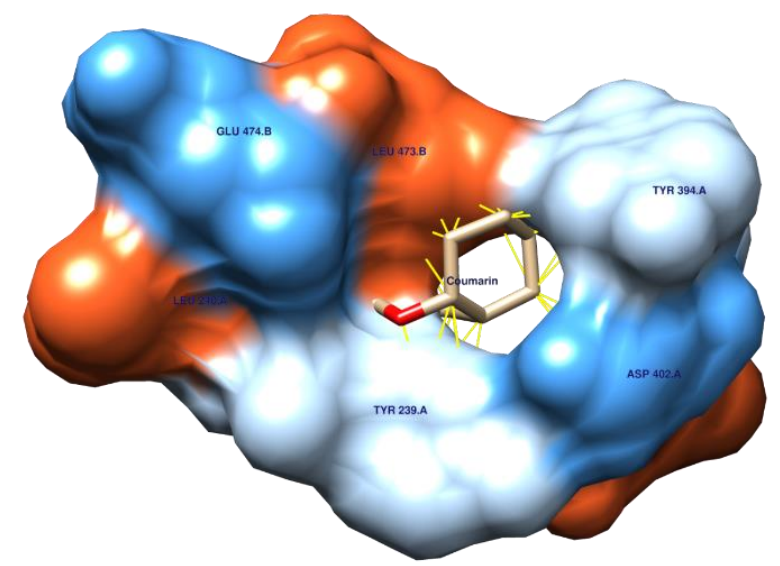

Figure 9. Coumarin bound to anthranilate synthase component II binding pocket residues.

\subsection{Toxicity profile of coumarin.}

Coumarin was found to have a water solubility value of $-2.753 \log S$, plasma protein binding value of 0.865 , acute oral toxicity of $2.514 \mathrm{Kg} / \mathrm{mol}$, and tetrahymena pyriformis toxicity value at $2.026 \mu \mathrm{g} / \mathrm{L}$ pIGC50.

3.7. In vitro anti-hemolytic property of coumarin.

Since coumarin makes a potential drug candidate for treating $S$. pneumoniae infection through inhibition of anthranilate synthase component II, the ability of coumarin to prevent the infection of $S$. pneumoniae through hemolysis was analyzed using the anti-hemolytic activity of coumarin. It was found that coumarin decreases the hemolysis of RBCs by $20 \%$ upon infection with S. pneumoniae-D39 in comparison to the untreated S. pneumoniae infection model where $S$. pneumoniae causes $90 \%$ RBC lysis. These results indicate that coumarin may act as a protectant in the host system against $S$. pneumoniae.

\section{Conclusions}

Coumarin, an aromatic compound, can be used as a structural analog for tryptophan to inhibit anthranilate synthase component II, a key enzyme in immune suppression during inflammation, thereby causing functional loss of the enzyme, aiding in preventing immune evasion by the organism.

\section{Funding}

This research received no external funding.

\section{Acknowledgments}

MCN and CS thank the JSS Academy of Higher Education \& Research for funding this work under the JSSAHER Student Research Grant 2019 (REG/DIR(R)/URG/54/2011-12). MCN, CS, and GS thank Glimetomics Bioresolve Private Limited for its enduring scientific support and aid in conducting this research study.

\section{Conflicts of Interest}

There is no conflict of interest among the authors whatsoever. 


\section{References}

1. Zivich, P.N.; Grabenstein, J.D.; Becker-Dreps, S.I.; Weber, D.J. Streptococcus pneumoniae outbreaks and implications for transmission and control: a systematic review. Pneumonia 2018, 10, 11, https://doi.org/10.1186/s41479-018-0055-4.

2. Cilloniz, C.; Martin-Loeches, I.; Garcia-Vidal, C.; San Jose, A.; Torres A. Microbial Etiology of Pneumonia: Epidemiology, Diagnosis and Resistance Patterns. International Journal of Molecular Sciences 2016, 17, https://doi.org/10.3390/ijms17122120.

3. Kaplan, S,L.; Mason, Jr. E.O. Management of infections due to antibiotic-resistant Streptococcus pneumoniae. Clin Microbiol Rev 1998, 11, 628-44, https://pubmed.ncbi.nlm.nih.gov/9767060.

4. Dion, C.F.; Ashurst, J.V. Streptococcus Pneumoniae. In Treasure Island 2020, https://pubmed.ncbi.nlm.nih.gov/29261971/.

5. Song, J.H.; Huh, K.; Chung, D.R.; Community-Acquired Pneumonia in the Asia-Pacific Region. Semin Respir Crit Care Med 2016, 37, 839-54, https://pubmed.ncbi.nlm.nih.gov/27960208.

6. Sheldon, A.T.J.; Antibiotic resistance: a survival strategy. Clin Lab Sci. 2005, 18, 170-80, https://pubmed.ncbi.nlm.nih.gov/16134477/.

7. Petchiappan, A.; Chatterji, D.; Antibiotic Resistance: Current Perspectives. ACS omega. 2017, 2, 7400-9, https://pubmed.ncbi.nlm.nih.gov/30023551/.

8. Musa, M.A.; Cooperwood, J.S.; Khan, M.O.F.; A review of coumarin derivatives in pharmacotherapy of breast cancer. Curr Med Chem. 2008, 15, 2664-79, https://pubmed.ncbi.nlm.nih.gov/18991629/.

9. Reen, F.J.; Gutiérrez-Barranquero, J.A.; Parages, M.L.; O Gara, F. Coumarin: a novel player in microbial quorum sensing and biofilm formation inhibition. Appl Microbiol Biotechnol 2018, 102, 2063-73, https://pubmed.ncbi.nlm.nih.gov/29392389.

10. Chitagudigi, M.N.; Shruthi, G.; Shivamallu, C. Molecular Interaction Studies for Inhibition of the Streptococcus pneumoniae Competence Stimulating Peptide (CSP1) by Potent Plant-Derived Compounds. Int. J. Life Sci. Pharma Res. 2020, 10(5), 154-166, http://dx.doi.org/10.22376/ijpbs/ijlpr.2020.10.5.P154-16.

11. Poumale, H.M.P.; Hamm, R.; Zang, Y.; Shiono, Y.; Kuete, V. 8 - Coumarins and Related Compounds from the Medicinal Plants of Africa. In: Kuete VBT-MPR in A, editor. Oxford: Elsevier 2013. 261-300, https://doi.org/10.1016/B978-0-12-405927-6.00008-4.

12. Matos, M.J.; Santana, L.; Uriarte, E.; Abreu, O.A.; Molina, E.;Yordi, E.G.; Coumarins - An Important Class of Phytochemicals. Phytochem - Isol Characterisation Role Hum Heal. 2015, https://doi.org/10.5772/59982.

13. Lee, J.H.; Kim, Y.G.; Cho, H.S.; Ryu, S.Y.; Cho, M.H.; Lee, J. Coumarins reduce biofilm formation and the virulence of Escherichia coli O157:H7. Phytomedicine 2014, 21, 1037-42, https://doi.org/10.1016/j.phymed.2014.04.008.

14. da Cunha, M.G.; de Cássia Orlandi; Sardi, J.; Freires, I.A.; Franchin, M.; Rosalen P.L.; Antimicrobial, antiadherence and antibiofilm activity against Staphylococcus aureus of a 4-phenyl coumarin derivative isolated from Brazilian geopropolis. Microb Pathog $2020, \quad 139, \quad 103855$, https://doi.org/10.1016/j.micpath.2019.103855.

15. Bortolotti, P.; Hennart, B.; Thieffry, C.; Jausions, G.; Faure, E.; Grandjean, T.; et all. Tryptophan catabolism in Pseudomonas aeruginosa and potential for inter-kingdom relationship. BMC Microbiol 2016, 16, 137, https://doi.org/10.1186/s12866-016-0756-x.

16. Lott, J.S. The tryptophan biosynthetic pathway is essential for Mycobacterium tuberculosis to cause disease. Biochem Soc Trans 2020, 48, 2029-37. https://doi.org/10.1042/BST20200194.

17. Zhang, P.; Jin, T.; Sahu, S.K.; Xu, J.; Shi, Q.; Liu, H.; et all. The distribution of tryptophan-dependent indole3-acetic acid synthesis pathways in bacteria unraveled by large-scale genomic analysis. Molecules 2019, 24, 1-14, https://doi.org/10.3390/molecules24071411.

18. Roager, H.M.; Licht, T.R.; Microbial tryptophan catabolites in health and disease. Nat Commun 2018, 9 , 3294, https://doi.org/10.1038/s41467-018-05470-4.

19. Sorgdrager, F.J.H.; Naudé, P.J.W.; Kema, I..P.; Nollen, E.A.; Deyn, P.P. De. Tryptophan Metabolism in Inflammaging: From Biomarker to Therapeutic Target. Frontiers in Immunology 2019, 10, 2565, https://doi.org/10.3389/fimmu.2019.02565.

20. Senggunprai, L.; Yoshinari, K.; Yamazoe, Y. Inhibitory effects of kynurenic acid, a tryptophan metabolite, and its derivatives on cytosolic sulfotransferases. Biochem J. 2009, 422, 455-62, https://doi.org/10.1042/BJ20090168. 
21. Zummo, F.P.; Marineo, S.; Pace, A. Civiletti, F.; Giardina, A.; Puglia, A.M. Tryptophan catabolism via kynurenine production in Streptomyces coelicolor: identification of three genes coding for the enzymes of tryptophan to anthranilate pathway. Appl Microbiol Biotechnol. 2012, 94, 719-28, https://doi.org/10.1007/s00253-011-3833-y.

22. Parthasarathy, A.; Cross, P.J.; Dobson, R.C.J.; Adams, L.E.; Savka, M.A.; Hudson, A.O. A Three-Ring Circus: Metabolism of the Three Proteogenic Aromatic Amino Acids and Their Role in the Health of Plants and Animals. Frontiers in Molecular Biosciences 2018, 5, 29, https://www.frontiersin.org/article/10.3389/fmolb.2018.00029.

23. Wong, R.S.Y. Role of Nonsteroidal Anti-Inflammatory Drugs (NSAIDs) in Cancer Prevention and Cancer Promotion. Adv Pharmacol Sci. 2019, 2019, https://doi.org/10.1155/2019/3418975.

24. Johnson, M.; Zaretskaya, I.; Raytselis, Y.; Merezhuk, Y.; McGinnis, S.; Madden, T.L. NCBI BLAST: a better web interface. Nucleic Acids Res. 2008, 36, 5-9, https://doi.org/10.1093/nar/gkn201.

25. Morgulis, A.; Coulouris, G.; Raytselis, Y.; Madden,T.L.; Agarwala, R.; Schäffer, A.A. Database indexing for production MegaBLAST searches. Bioinformatics. 2008, 24, 1757-64, https://doi.org/10.1093/bioinformatics/btn322.

26. Papadopoulos, J.S.; Agarwala, R. COBALT: constraint-based alignment tool for multiple protein sequences. Bioinformatics. 2007, 23, 1073-9, https://doi.org/10.1093/bioinformatics/btm076.

27. Kumar, S.; Stecher, G.; Li, M.; Knyaz, C.; Tamura, K. MEGA X.: Molecular evolutionary genetics analysis across computing platforms. Mol Biol Evol. 2018, 35, 1547-9, https://doi.org/10.1093/molbev/msy096.

28. Kumar, S.; Tamura, K.; Jakobsen, I.B.; Nei, M. MEGA2: Molecular evolutionary genetics analysis software. Bioinformatics. 2002, 17, 1244-5, https://doi.org/10.1093/bioinformatics/17.12.1244.

29. Hall, B.G. Building phylogenetic trees from molecular data with MEGA. Mol Biol Evol. 2013, 30, 1229-35, https://doi.org/10.1093/molbev/mst012.

30. De Moraes Russo, C.A.; Selvatti, A.P. Bootstrap and rogue identification tests for phylogenetic analyses. Mol Biol Evol. 2018, 35, 2327-33, https://doi.org/10.1093/molbev/msy118.

31. Sippl, M.J. Recognition of errors in three-dimensional structures of proteins. Proteins: Structure, Function, and Bioinformatics. 1993, 17, 355-62, https://doi.org/10.1002/prot.340170404.

32. Al-Obaidi, A.; Elmezayen, A.D.; Yelekçi, K. Homology modeling of human GABA-AT and devise some novel and potent inhibitors via computer-aided drug design techniques. J Biomol Struct Dyn. 2020, 28, 1-11, https://doi.org/10.1080/07391102.2020.1774417.

33. Heo, L.; Park, H.; Seok, C. GalaxyRefine: Protein structure refinement driven by side-chain repacking. Nucleic Acids Res. 2013, 41, 384-8, https://doi.org/10.1093/nar/gkt458.

34. Spessard, G.O. ACD Labs/LogP dB 3.5 and ChemSketch 3.5. J Chem Inf Comput Sci. 1998, https://www.semanticscholar.org/paper/ACD-Labs\%2FLogP-dB-3.5-and-ChemSketch-3.5Spessard/8be1d08824bd503c3e8f5697dc751fc9c7efdd57.

35. Bursulaya, B.D.; Totrov, M.; Abagyan, R.; Brooks, C.L. Comparative study of several algorithms for flexible ligand docking. J Comput Aided Mol Des. 2003, https://doi.org/10.1023/b:jcam.0000017496.76572.6f.

36. Morris, G.M.; Dallakyan, S. AutoDock - AutoDock. 2013, http://autodock.scripps.edu/.

37. Forli, W.; Halliday, S.; Belew, R.; Olson, A. AutoDock Version 4.2. Citeseer. 2012, http://autodock.scripps.edu/faqs-help/manual/autodock-4-2-user-guide/AutoDock4.2_UserGuide.pdf.

38. Pettersen, E.F.; Goddard, T.D.; Huang, C.C.; Couch, G.S.; Greenblatt, D.M.; Meng, E.C.; UCSF Chimera--a visualization system for exploratory research and analysis. J Comput Chem. 2004, 25, 1605-12, https://doi.org/10.1002/jcc.20084.

39. Dong, J.; Wang, N-N.; Yao, Z-J.; Zhang, L.; Cheng, Y.; Ouyang, D.; ADMETlab: a platform for systematic ADMET evaluation based on a comprehensively collected ADMET database. J Cheminform 2018, 10, 29, https://doi.org/10.1186/s13321-018-0283-X.

40. Fan, D.; Yang, H.; Li, F.; Sun, L.; Di, P.; Li, W. In silico prediction of chemical genotoxicity using machine learning methods and structural alerts. Toxicol Res (Camb) 2018, 7, 211-220, https://doi.org/10.1039/c7tx00259a.

41. Daina, A.; Michielin, O.; Zoete, V. SwissADME: a free web tool to evaluate pharmacokinetics, drug-likeness and medicinal chemistry friendliness of small molecules. Sci Rep 2017, 7, 42717, https://doi.org/10.1038/srep42717.

42. Shruthi, G.; Prasad, K.S.; Vinod, T.P.; Balamurugan, V.; Shivamallu, C. Green Synthesis of Biologically Active Silver Nanoparticles through a Phyto-Mediated Approach Using Areca catechu Leaf Extract. ChemistrySelect. 2017, 2, https://doi.org/10.1002/slct.201702257. 